\title{
Exercise Training for Heart Failure Patients with and without Systolic Dysfunction: An Evidence-Based Analysis of How Patients Benefit
}

\author{
Neil Smart \\ Faculty of Health Science and Medicine, Bond University, Robina, QLD 4229, Australia \\ Correspondence should be addressed to Neil Smart, nsmart@bond.edu.au \\ Received 23 July 2010; Accepted 19 September 2010 \\ Academic Editor: Javed Butler \\ Copyright () 2011 Neil Smart. This is an open access article distributed under the Creative Commons Attribution License, which \\ permits unrestricted use, distribution, and reproduction in any medium, provided the original work is properly cited.

\begin{abstract}
Significant benefits can be derived by heart failure patients from exercise training. This paper provides an evidence-based assessment of expected clinical benefits of exercise training for heart failure patients. Meta-analyses and randomized, controlled trials of exercise training in heart failure patients were reviewed from a search of PubMed, Cochrane Controlled Trial Registry (CCTR), CINAHL, and EMBASE. Exercise training improves functional capacity, quality of life, hospitalization, and systolic and diastolic function in heart failure patients. Heart failure patients with preserved systolic function (HFnEF) participating in exercise training studies are more likely to be women and are 5-7 years older than their systolic heart failure (CHF) counterparts. All patients exhibit low functional capacities, although in HFnEF patients this may be age related, therefore subtle differences in exercise prescriptions are required. Published works report that exercise training is beneficial for heart failure patients with and without systolic dysfunction.
\end{abstract}

\section{Background}

Exercise training has become an accepted adjunct therapy for patients with systolic heart failure. Recent data have shown that exercise is considered beneficial for heart failure patients in terms of improved mortality and morbidity [1], quality of life [2,3], functional capacity [4], and improved cardiac [5] and vascular function [6]. Characteristics of heart failure patients who participate in community-based prevalence and exercise training studies may be different with the latter likely to be younger and predominantly male $[1,4,7,8]$. North American [9], European [10], and Australian [11] guidelines on the use of exercise training in heart failure patients have been developed, although subtle differences exist between the various guidelines. Subtle differences may also exist in the demographics of heart failure patients with abnormal systolic function (CHF) and heart failure patients with normal left ventricular ejection fraction (HFnEF). CHF patients are normally defined as having left ventricular ejection fraction $<50 \%$ and impaired diastolic filling, while HFnEF patients have a normal $(>50 \%)$ ejection fraction but impaired diastolic filling.
The aims of this work were to review highlighted differences between the demographics of heart failure patients participating in prevalence studies and those completing exercise training studies. Moreover demographic data were presented for systolic heart failure patients and those with heart failure with preserved systolic function. This work also aimed to provide a clinical update on the benefits of exercise training in heart failure patients.

\section{Methods}

Data relating to exercise training protocols in heart failure patients and subsequent outcomes were reviewed. Pub Med (MEDLINE), Medscape, EMBASE, CINAHL, and Cochrane Controlled Trials Registry were searched up until June 2010. Reference lists of papers found were scrutinized for new references. North American, European, and Australian heart failure treatment guidelines were also sourced. Search terms used were combinations of the terms exercise training, heart failure, left ventricular dysfunction, physical training, resistance training, guidelines, position stands, systematic reviews, 


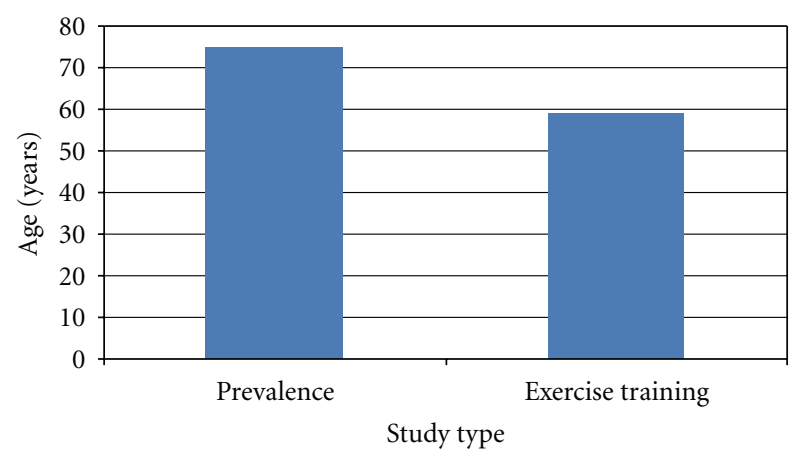

FIgURE 1: Age comparison of heart failure patients in both prevalence and exercise studies.

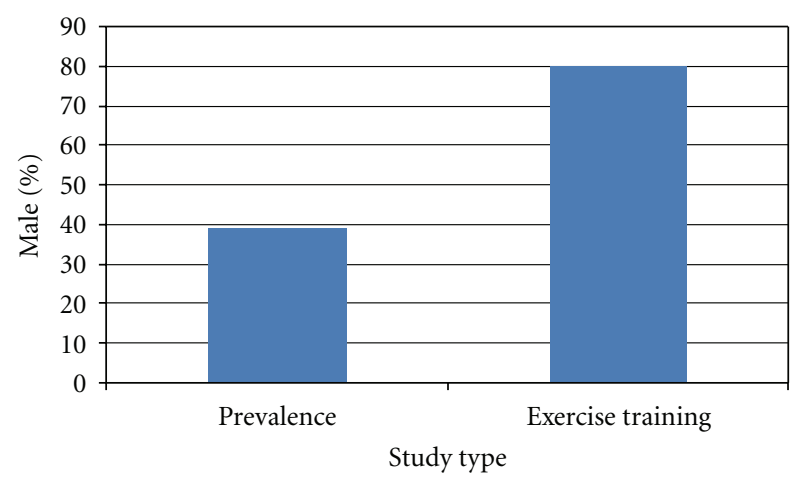

FIGURE 2: Gender comparison of heart failure patients in both prevalence and exercise studies.

meta-analysis, quality of life, functional capacity, mortality, morbidity, cardiac function, heart failure with preserved systolic function, safety, cytokine expression, brain natriuretic peptide, and aerobic exercise. There were no restrictions on the year of publication. We examined the latest editions of relevant journals that were not yet available on electronic databases. The reference lists of identified articles were subsequently scrutinized and relevant articles were included. Information on clinical variables was archived in a database. Communitybased heart failure prevalence studies were also sought to draw comparisons with exercise studies in heart failure patients.

Outcome measures fell into three categories: demographics, clinical measures, and public health issues. Demographics: age and gender of heart failure patients enrolled in community-based prevalence versus exercise training studies. Clinical measures: mortality and morbidity rates, functional capacity, quality of life, cardiac function, cytokine, and brain natriuretic peptide (BNP) expression in those who did and did not undertake exercise training. Public Health: number needed to treat, cost-effectiveness, safety, and minimization of adverse events during exercise training.

\section{Results}

3.1. Demographics of Heart Failure Patients in both the Community and in Exercise Training Studies.. Heart failure patients who participate in exercise training research tend to be younger (Figure 1) and twice as likely to be male (Figure 2) compared to those heart failure patients studied in prevalence research $[1,12]$; the latter appear to provide a better representation of heart failure patients in the community, suggesting exercise studies may be prone to participant selection bias.

Evidence suggests younger, male heart failure patients are more likely to participate in exercise training due to possible selection bias.

\subsection{Demographics of CHF and HFnEF Patients Participating} in Exercise Training Studies.. Data from exercise training studies of heart failure patients [4] reports CHF patients are younger, mean $59 \pm 7$ years, and more likely to be male $(79 \%)$ compared to HFnEF patients, mean age $65 \pm 5$ years and $50 \%$ male.

Evidence suggests CHF patients participating in exercise training are younger and more likely to be male.

\subsection{Clinical or Prognostic Characteristics}

3.3.1. Mortality and Hospitalization Benefits. A meta-analysis of exercise training programs in heart failure patients (ExTraMATCH) reported benefits in terms of mortality and hospitalization [1]. A subanalysis of this study revealed the reduction in overall and cardiac mortality and hospitalization appears to be more likely in sicker patients, specifically those with lower left ventricular ejection fraction, New York Heart Association class III/IV, ischemic cardiomyopathy, lower peak $\mathrm{VO}_{2}$, and those over 60 years of age. In terms of these exercise training program characteristics, these mortality benefits appear to be more apparent in those patients that adhere to an exercise training program for 28 weeks or more [1]. All studies included in the ExTraMATCH meta-analysis used cycling as the training modality. In comparison the largest (2,300 patients) randomized, controlled study of exercise training in heart failure patients to date, the heart failure ACTION (HF-ACTION) study [8], reported nonsignificant reductions in mortality and hospitalization, especially in those who do not have factors highly prognostic of mortality. Specifically, duration of baseline cardiopulmonary exercise testing, left ventricular ejection fraction, Beck Depression Inventory II score, and history of atrial fibrillation or flutter were identified as highly prognostic of the primary endpoint of all-cause mortality or hospitalization. After adjusting for prognostic hence exercise training was found to reduce the incidence of all-cause mortality or all-cause hospitalization by $11 \%$. The data from ExTraMATCH and HF-ACTION appear to contrast one another, while the former showed a mortality benefit that was most obvious in the sickest patients, the latter only showed a mortality benefit once the patients with prognostic symptoms had been removed from the analysis. 
Evidence equivocal for mortality and hospitalization benefits and also whether disease severity predicts which patients may benefit most.

3.3.2. Functional Capacity. Meta-analysis reported that heart failure patients can typically expect to improve their functional capacity by $15 \%-17 \%$ following exercise training [4] and this is a powerful predictor of prognosis in this population [13]. Peak $\mathrm{VO}_{2}$ values $>13-14 \mathrm{~mL} / \mathrm{kg} / \mathrm{min}$ may confer a $50 \%$ cardiac mortality reduction, while a $1 \mathrm{~mL} / \mathrm{kg} / \mathrm{min}$ increase may be associated with a $10 \%$ lower cardiac mortality [14]. Peak $\mathrm{VO}_{2}$ changes appear to be independent of the type of exercise training undertaken, for example, aerobic, intermittent or resistance training [4]. In comparison, the (HF-ACTION) study [8] reported only a $4 \%$ improvement in peak $\mathrm{VO}_{2}$. Meta-analysis data was generated from generally smaller, single site studies which often had facilitated good short-term exercise training adherence rates. HF-ACTION trial authors reported poor adherence in the early part of the training program, and the multicentre study design may have affected the magnitude of change in peak $\mathrm{VO}_{2}$. Moreover, the meta-analysis data was generated mainly from short $(<3$ month) duration studies with only two studies $>12$ months duration [15, 16], while HF-ACTION reported both 3 month and 12 month data. Twelve months is a difficult period to retain patients, due to either attrition, illness, or death, and also maintain exercise adherence in those who do not withdraw from the study. It has been suggested that exercise training studies tend to overestimate the size of change in peak $\mathrm{VO}_{2}$ of exercise training participants [17], due to variations in patient motivation and the impracticality of blinding exercise training subjects. The more robust design of HF-ACTION may have minimized the overestimation of peak $\mathrm{VO}_{2}$ and therefore changes were smaller, but perhaps more reliable. Only one published exercise training study in HFnEF has provided functional capacity data to date suggesting the size of the change in peak $\mathrm{VO}_{2}$ may be similar, or even greater, to that seen in CHF patients.

Evidence suggest functional capacity undoubtedly improves, but what is uncertain is the size of change in Peak $\mathrm{VO}_{2}$ which is likely to be between $4 \%-15 \%$.

3.3.3. Quality of Life (QOL). In a systematic review of exercise training in heart failure patients, QOL assessment was improved in 11 of 16 randomized controlled studies [2]. A more recent meta-analysis of 35 randomized controlled trials, reported that heart failure patients can expect an improvement of nearly 10 points in Minnesota living with heart Failure Questionnaire scores following exercise training [3].

Evidence is unequivocal for improvement in quality of life.

3.3.4. Cardiac (Systolic) Function. A recent meta-analysis [5] showed that exercise training improved left ventricular ejection fraction (LVEF), end-diastolic volume (EDV), and end-systolic volume (ESV) in patients with heart failure.
The weighted mean difference (WMD) of LVEF was significantly improved after aerobic exercise training ( 9 trials, 538 patients, $\mathrm{WMD}=2.59 \%$; $95 \%$ confidence interval $(\mathrm{CI})$ $1.44 \%$ to $3.74 \%$ ), EDV (371 patients; $\mathrm{WMD}=-11.49 \mathrm{~mL}$; 95\% CI -19.95 to $-3.02 \mathrm{~mL}$ ), and ESV (371 patients; WMD $=-12.87 \mathrm{~mL} ; 95 \% \mathrm{CI}-17.80$ to $-7.93 \mathrm{~mL}$. The authors concluded that aerobic training, but not combined aerobic and strength training, or isolated strength training reverse LV remodelling in clinically stable individuals with heart failure. Other data suggest that high intensity (95\% peak heart rate) aerobic interval walking will confer even greater improvements in systolic function (baseline LVEF 28\%, posttraining LVEF 38\%) [18].

Evidence suggests exercise training will improve systolic function and should be predominantly aerobic, and if tolerated, intermittent high intensity, in nature as this will convey the greatest improvements in systolic function.

3.4. Cardiac (Diastolic) Function. Published randomized, controlled trials of exercise training in heart failure patients with outcome measures of diastolic function, assessed by tissue Doppler have reported variable results. The work of Belardinelli [15] showed improvements in isovolumic relaxation time (ms), early and late diastolic filling in a 2-month exercise training study of heart failure patients with moderate-severe systolic function. Similarly improved left ventricular diastolic stiffness [19] and reduced filling pressure (E/Ea ratio) [18] in heart failure patients has been reported in two other randomized, controlled trials of exercise training.

Evidence suggests exercise training may enhance diastolic function.

3.5. Circulating Levels of Proinflammatory Cytokines. Several small randomized controlled trials have conducted analyses of the effect of different forms of exercise training (high versus low intensity, cycling versus electrical stimulation, or respiratory muscle training) on serum measures of proinflammatory cytokines in patients with congestive heart failure. Interleukin-6 (IL-6) and tumour necrosis factoralpha are the two most commonly studied proinflammatory cytokines [20-27]. A recent, elegant review clarified the role of IL-6 as a fuel (glucose) sensor [28]. As glucose metabolism may be affected in heart failure patients, especially during exercise Pederson's review may explain some of the varied effects of exercise training on IL-6.

Evidence-equivocal for reducing systemic levels of inflammatory cytokines.

3.6. Brain Natriuretic Peptide (BNP) Levels. BNP levels are also raised in the circulation of patients with chronic heart failure (CHF) and may be related to disease severity or poor management [29]. B-type natriuretic peptide (BNP) is a cardiac neurohormone released as pre-pro-BNP and then enzymatically cleaved to $\mathrm{N}$-terminal-proBNP and BNP 


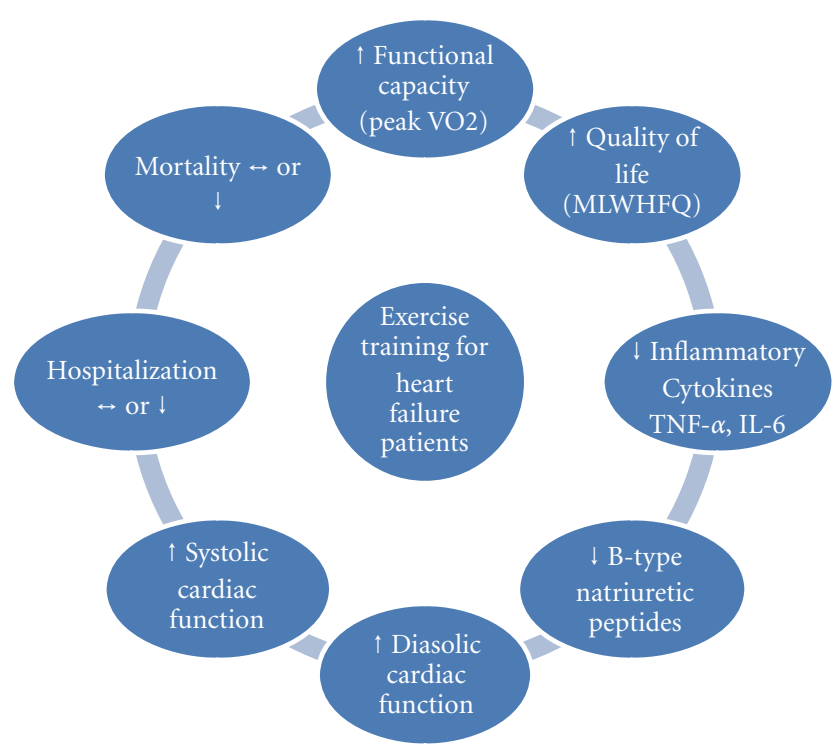

FIgURE 3: Clinical or prognostic markers of change in heart failure patients undertaking exercise training.

upon ventricular myocyte stretch. Blood measurements of BNP have been used to identify patients with heart failure $[29,30]$. Currently, BNP assay is used as a diagnostic and prognostic aid in congestive heart failure. In general, a BNP level $<100 \mathrm{pg} / \mathrm{mL}$ excludes acutely decompensated heart failure [29]. A recent systematic review in order to establish whether exercise training of CHF patients has an effect on circulating BNP levels [31]. Meta-analysis of randomized, controlled trials showed that exercise training had a favourable effect on BNP $-79 \mathrm{pg} / \mathrm{mL} 95 \%$ C.I. ( -141 to -17) $P=.01$ and NT-proBNP $-621 \mathrm{pg} / \mathrm{mL} 95 \%$ C.I. $(-844$ to -398$) P<.00001$. Two of the included studies had low weekly energy expenditure $(<400 \mathrm{Kcal} /$ week $)$ and changes in BNP and NT-pro-BNP were not significant in these studies $[32,33]$. Patients who did not undergo training showed no changes, suggesting blunting of adrenergic overactivity and of natriuretic peptide overexpression [31].

Evidence unequivocal that B-type natriuretic peptides are reduced following exercise training, moreover a minimum weekly energy expenditure (circa $400 \mathrm{Kcal} \cdot$ week $^{-1}$ ) may be required to elicit changes in $B$-type natriuretic peptides.

A summary of clinical markers of heart failure prognosis can be seen in Figure 3.

\subsection{Public Health Issues}

3.7.1. Number Needed to Teat (NNT) to Prevent 1 Death. As peak $\mathrm{VO}_{2}$ is a strong predictor of prognosis [13] and heart failure patients undertaking exercise training may expect a $4 \%-15 \%$ improvement in peak $\mathrm{VO}_{2}$. An associated mortality benefit has been reported $[1,15]$, although HF-ACTION data suggest these benefits remain equivocal. A systematic review calculated the number of heart failure patients that need to undertake exercise training in order to prevent one death is 17 over two years [1]. This value corresponds to 34 patients over a 12 month period.

Evidence suggests that 34 heart failure patients need to be provided with exercise training for 12 months to prevent one death from exercise training over this period.

3.7.2. Cost-Effectiveness. Cost of treatment will always be a consideration in clinical decision making. Table 1 describes the estimated cost-effectiveness of three therapies for heart failure. To highlight the example the cost has been calculated for conducting a heart failure specific exercise program in Australia (in mid-2010), in both a supervised hospital setting and home-based setting (Table 1). A salary of AUS\$ $60 \mathrm{~K}$ p.a. for a full-time graduate exercise physiologist is typical, and would be required to manage 34 patients, however start-up equipment costs of AUS\$ 5-10 K have not been included in the estimation as these and other ongoing costs have been excluded. A home exercise program may require only 1 day per week of the same exercise physiologist to telephone patients to verify progress, but little or no equipment-the next section addresses home exercise in more detail. We have used examples cited on the University of Toronto's evidencebased medicine website [34] of trials of a beta-1 selective blocker [35] and the diuretic Aldactone [36]. When we compare treatment costs to prevent mortality in one patient, we can see that hospital-based exercise is comparable in cost to beta-blockade but noticeably less cost-effective than Aldactone, while home exercise training is clearly more costeffective than beta-blockade but not Aldactone.

Evidence suggests exercise training, especially homebased, is a cost effective adjunct treatment.

3.7.3. Safety. Exercise may increase the risk of sudden cardiac death [38]. While both exercise training and the heart failure condition each convey an increased risk of sudden death or adverse event, the published works in this field are yet to identify as single death that could be directly linked to exercise exertion in over 100,00 patient-hours of exercise training [4]. For this reason odds ratios for heart failure patients have not been calculated. Data from the HFACTION trial were zero deaths per 1,000 exercise tests and 0.45 nonfatal major CV events per 1,000 exercise tests ( $95 \%$ CI 0.11-1.81). Recent data suggest that although, sudden cardiac death (SCD) and cardiac events are more prevalent in the morning hours, data do not exist to suggest the time of day exercise training is conducted may not increase the risk of SCD or other adverse events [38].

Supervised outpatient trials may proffer better adherence rates than home exercise, possibly due to poor motivation and concerns about safety [39]. Strategies that improve home exercise compliance require regular patient contact and methods of verifying patient adherence. Nevertheless it is possible to sustain peak $\mathrm{VO}_{2}$ and quality of life (QOL) in a "Home" exercise program 12 months after outpatient cardiac rehabilitation discharge $[16,40]$. A recent systematic review 
TABLE 1: Relative costs of various treatments for one mortality avoided.

\begin{tabular}{lcc}
\hline Treatment & Number Needed to Treat per annum & Estimated Cost (AUS\$) per annum \\
\hline Beta-Blockade [35] & 72 & $65 \mathrm{~K}$ \\
Aldactone (Spironolactone) [36] & 4.5 & $5 \mathrm{~K}$ \\
Hospital based exercise training [37] & 34 & $60 \mathrm{~K}$ \\
Home based exercise training & N/A & $12 \mathrm{~K}$ \\
\hline
\end{tabular}

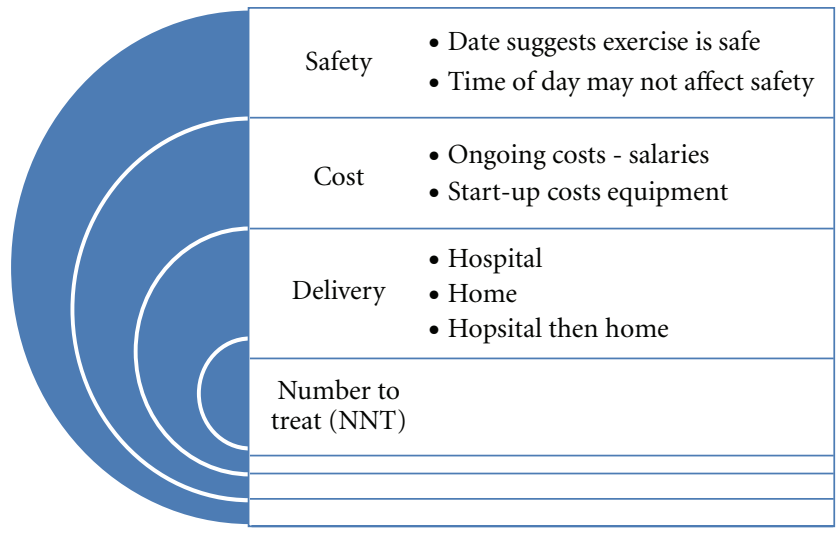

Figure 4: Public health issues associated with exercise training programs for heart failure patients.

reported that home exercise for heart failure patients may elicit similar magnitude of change in peak $\mathrm{VO}_{2}$ compared to hospital based programs, although some of the data was generated from combined hospital and home exercise studies [40]. A tailored exercise program may optimise health benefits while minimising risk of exertional events. Periodic measurement of functional capacity, either directly or indirectly may protect against under- or overestimating the optimal training intensity.

Evidence suggests exercise training is safe for heart failure patients, but home exercise is most beneficial when it follows a supervised, hospital-based program.

A summary of public health issues related to heart failure exercise training can be seen in Figure 4.

\section{Discussion}

A chronological history of exercise training for heart failure patients makes interesting reading. Prior to the 1990's the advice almost unequivocally given to heart failure patients was to avoid physical exertion. A handful of studies prior to the 1990's suggested exercise training was safe and possibly beneficial in reducing dyspnea and improving mood. In the 1990's and 2000's numerous studies and several metaanalyses have highlighted the importance of regular physical activity in improving not only improving physical fitness and quality of life, but also cardiac and blood vessel function and systemic inflammatory and neurohormonal markers of heart failure. Moreover exercise appears to be safe and most people with heart failure, with the exception of New York Heart Association class IV and some other high risk-heart failure patient categories, should be advised to regularly exercise.

The change in consensus of opinion towards safety and efficacy of exercise training was based upon data synthesis from many small- to medium-sized trials. Notably, metaanalysis [1] had suggested significant mortality and hospitalization benefits could be derived from exercise training heart failure patients, however closer analysis of the data suggested statistical significance of mortality and hospitalization was absent if the study of Belardinelli [15] was removed from the meta-analysis. Then, in late 2008 data was released from the HF-ACTION study [8] the largest (2,300 patients) exercise training study of heart failure patients to date. HFACTION reported nonsignificant reductions in mortality and hospitalization. After adjusting for highly prognostic predictors, exercise training was associated with modest significant reductions for both mortality and heart failure hospitalization. These findings together with the cost and efficacy data presented in this paper suggest exercise has a beneficial role in the nonpharmacological management of heart failure.

\section{Conclusions}

Differences exist in the demographics of participants of prevalence and exercise training studies in heart failure patients. Exercise training is safe, effective, and not cost prohibitive. Evidence shows exercise training-induced improvements are possible in many indicators of heart failure patient prognosis; functional capacity, quality of life, systolic and diastolic function, BNP and NT-pro-BNP expression. Nevertheless it remains questionable whether exercise training reduces cardiac deaths and hospitalisation and whether disease severity predicts which patients are most likely to benefit.

\section{References}

[1] M. F. Piepoli, C. Davos, D. P. Francis, and A. J. Coats, "Exercise training meta-analysis of trials in patients with chronic heart failure (ExTraMATCH)," British Medical Journal, vol. 328, article 189, 2004.

[2] F. Lloyd-Williams, F. S. Mair, and M. Leitner, "Exercise training and heart failure: a systematic review of current evidence," British Journal of General Practice, vol. 52, no. 474, pp. 47-55, 2002. 
[3] B. A. F. van Tol, R. J. Huijsmans, D. W. Kroon, M. Schothorst, and G. Kwakkel, "Effects of exercise training on cardiac performance, exercise capacity and quality of life in patients with heart failure: a meta-analysis," European Journal of Heart Failure, vol. 8, no. 8, pp. 841-850, 2006.

[4] N. Smart and T. H. Marwick, "Exercise training for patients with heart failure: a systematic review of factors that improve mortality and morbidity," American Journal of Medicine, vol. 116, no. 10, pp. 693-706, 2004.

[5] M. J. Haykowsky, Y. Liang, D. Pechter, L. W. Jones, F. A. McAlister, and A. M. Clark, "A meta-analysis of the effect of exercise training on left ventricular remodeling in heart failure patients. The benefit depends on the type of training performed," Journal of the American College of Cardiology, vol. 49, no. 24, pp. 2329-2336, 2007.

[6] R. Hambrecht, L. Hilbrich, S. Erbs et al., "Correction of endothelial dysfunction in chronic heart failure: additional effects of exercise training and oral L-arginine supplementation," Journal of the American College of Cardiology, vol. 35, no. 3, pp. 706-713, 2000.

[7] G. S. Bleumink, A. M. Knetsch, M. C. J. M. Sturkenboom et al., "Quantifying the heart failure epidemic: prevalence, incidence rate, lifetime risk and prognosis of heart failurethe Rotterdam Study," European Heart Journal, vol. 25, no. 18, pp. 1614-1619, 2004.

[8] C. M. O'Connor, D. J. Whellan, K. L. Lee et al., "Efficacy and safety of exercise training in patients with chronic heart failure HF-ACTION randomized controlled trial," Journal of the American Medical Association, vol. 301, no. 14, pp. 14391450, 2009.

[9] S. A. Hunt, D. W. Baker, M. H. Chin et al., "ACC/AHA guidelines for the evaluation and management of chronic heart failure in the adult: executive summary. A report of the American College of Cardiology/American Heart Association Task Force on Practice Guidelines (Committee to Revise the 1995 Guidelines for the Evaluation and Management of Heart Failure)," Journal of the American College of Cardiology, vol. 38, no. 7, pp. 2101-2113, 2001.

[10] P. Giannuzzi, "Recommendations for exercise training in chronic heart failure patients," European Heart Journal, vol. 22, no. 2, pp. 125-135, 2001.

[11] A. Tonkin, P. Barter, J. Best et al., "National heart foundation of Australia and the cardiac society of Australia and New Zealand: position statement on lipid management-2005," Heart, Lung \& Circulation, vol. 14, no. 4, pp. 275-291, 2005.

[12] E. F. Philbin, T. A. Rocco Jr., N. W. Lindenmuth, K. Ulrich, and P. L. Jenkins, "Systolic versus diastolic heart failure in community practice: clinical features, outcomes, and the use of angiotensin-converting enzyme inhibitors," American Journal of Medicine, vol. 109, no. 8, pp. 605-613, 2000.

[13] D. Mancini, T. LeJemtel, and K. Aaronson, "Peak VO2: a simple yet enduring standard," Circulation, vol. 101, no. 10, pp. 1080-1082, 2000.

[14] T. Kavanagh, D. J. Mertens, L. F. Hamm et al., "Peak oxygen intake and cardiac mortality in women referred for cardiac rehabilitation," Journal of the American College of Cardiology, vol. 42, no. 12, pp. 2139-2143, 2003.

[15] R. Belardinelli, D. Georgiou, G. Cianci, and A. Purcaro, "Randomized, controlled trial of long-term moderate exercise training in chronic heart failure: effects on functional capacity, quality of life, and clinical outcome," Circulation, vol. 99, no. 9, pp. 1173-1182, 1999.
[16] R. S. McKelvie, K. K. Teo, R. Roberts et al., "Effects of exercise training in patients with heart failure: the Exercise Rehabilitation Trial (EXERT)," American Heart Journal, vol. 144, no. 1, pp. 23-30, 2002.

[17] T. Meyer, J. Scharhag, and W. Kindermann, "Peak oxygen uptake: myth and truth about an internationally accepted reference value," Zeitschrift fur Kardiologie, vol. 94, no. 4, pp. 255-264, 2005.

[18] U. Wisløff, A. Støylen, J. P. Loennechen et al., "Superior cardiovascular effect of aerobic interval training versus moderate continuous training in heart failure patients: a randomized study," Circulation, vol. 115, no. 24, pp. 3086-3094, 2007.

[19] G. Malfatto, G. Branzi, G. Osculati et al., "Improvement in left ventricular diastolic stiffness induced by physical training in patients with dilated cardiomyopathy," Journal of Cardiac Failure, vol. 15, no. 4, pp. 327-333, 2009.

[20] S. Adamopoulos, A. J. S. Coats, F. Brunotte et al., "Physical training improves skeletal muscle metabolism in patients with chronic heart failure," Journal of the American College of Cardiology, vol. 21, no. 5, pp. 1101-1106, 1993.

[21] S. Adamopoulos, J. Parissis, D. Karatzas et al., "Physical training modulates proinflammatory cytokines and the soluble Fas/soluble Fas ligand system in patients with chronic heart failure," Journal of the American College of Cardiology, vol. 39, no. 4, pp. 653-663, 2002.

[22] V. M. Conraads, P. Beckers, J. Bosmans et al., "Combined endurance/resistance training reduces plasma TNF- $\alpha$ receptor levels in patients with chronic heart failure and coronary artery disease," European Heart Journal, vol. 23, no. 23, pp. 1854-1860, 2002.

[23] A. Ferraz, E. A. Boochi, R. S. Meneghelo, I. I. Umeda, and N. Salvarane, "Sensitive C-reactive protein is reduced by exercise training in chronic heart failure patients: a prospective, randomized, controlled study," Circulation, vol. 110, pp. 793794, 2004.

[24] S. Gielen, V. Adams, S. Möbius-Winkler et al., "Antiinflammatory effects of exercise training in the skeletal muscle of patients with chronic heart failure," Journal of the American College of Cardiology, vol. 42, no. 5, pp. 861-868, 2003.

[25] A. I. Karavidas, K. G. Raisakis, J. T. Parissis et al., "Functional electrical stimulation improves endothelial function and reduces peripheral immune responses in patients with chronic heart failure," European Journal of Cardiovascular Prevention and Rehabilitation, vol. 13, no. 4, pp. 592-597, 2006.

[26] J. P. LeMaitre, S. Harris, K. A. A. Fox, and M. Denvir, "Change in circulating cytokines after 2 forms of exercise training in chronic stable heart failure," American Heart Journal, vol. 147, no. 1, pp. 100-105, 2004.

[27] D. Xu, B. Wang, Y. Hou, H. Hui, S. Meng, and Y. Liu, "The effects of exercise training on plasma tumor necrosis factoralpha, blood leucocyte and its components in congestive heart failure patients," Zhonghua Nei Ke Za Zhi, vol. 41, no. 4, pp. 237-240, 2002.

[28] B. K. Pedersen and M. A. Febbraio, "Muscle as an endocrine organ: focus on muscle-derived interleukin-6," Physiological Reviews, vol. 88, no. 4, pp. 1379-1406, 2008.

[29] P. A. McCullough, "B-type natriuretic peptide and its clinical implications in heart failure," The American heart hospital journal, vol. 2, no. 1, pp. 26-33, 2004.

[30] P. Jourdain, F. Funck, E. Canault et al., "Value of brain peptide in the management of suspected cardiac failure in the emergency room," Archives des Maladies du Coeur et des Vaisseaux, vol. 95, no. 9, pp. 763-767, 2002. 
[31] N. A. Smart and M. Steele, "Systematic review of the effect of aerobic and resistance exercise training on systemic brain natriuretic peptide (BNP) and N-terminal BNP expression in heart failure patients," International Journal of Cardiology, vol. 140 , pp. $260-265,2010$.

[32] J. A. Butterfield, S. C. Faddy, P. Davidson, and B. Ridge, "Exercise training in patients with stable chronic heart failure: effects on thoracic impedance cardiography and B-type natriuretic peptide," Journal of Cardiopulmonary Rehabilitation and Prevention, vol. 28, no. 1, pp. 33-37, 2008.

[33] S. Jónsdóttir, K. K. Andersen, A. F. Sigurosson, and S. B. Sigurosson, "The effect of physical training in chronic heart failure," European Journal of Heart Failure, vol. 8, no. 1, pp. 97-101, 2006.

[34] Uo. Toronto, "More on number needed to treat," 2008, http://www.cebm.net/index.aspx?o=1025.

[35] A. Hjalmarson, S. Goldstein, B. Fagerberg et al., "Effects of controlled-release metoprolol on total mortality, hospitalizations, and well-being in patients with heart failure: the metoprolol CR/XL randomized intervention trial in congestive heart failure (MERIT-HF)," Journal of the American Medical Association, vol. 283, no. 10, pp. 1295-1302, 2000.

[36] B. Pitt, F. Zannad, W. J. Remme et al., "The effect of spironolactone on morbidity and mortality in patients with severe heart failure," New England Journal of Medicine, vol. 341, no. 10, pp. 709-717, 1999.

[37] Website UoTEbm, "Glossary- moreon number needed to treat," 2008, http://www.cebm.net/index.aspx?o=1025.

[38] P. D. Thompson, B. A. Franklin, G. J. Balady et al., "Exercise and acute cardiovascular events: placing the risks into perspective a scientific statement from the American Heart Association Council on Nutrition, Physical Activity, and Metabolism and the Council on Clinical Cardiology," Circulation, vol. 115, no. 17, pp. 2358-2368, 2007.

[39] K. M. Smith, H. M. Arthur, R. S. McKelvie, and J. Kodis, "Differences in sustainability of exercise and health-related quality of life outcomes following home or hospital-based cardiac rehabilitation," European Journal of Cardiovascular Prevention and Rehabilitation, vol. 11, no. 4, pp. 313-319, 2004.

[40] R. Hwang and T. Marwick, "Efficacy of home-based exercise programmes for people with chronic heart failure: a metaanalysis," European Journal of Cardiovascular Prevention and Rehabilitation, vol. 16, no. 5, pp. 527-535, 2009. 


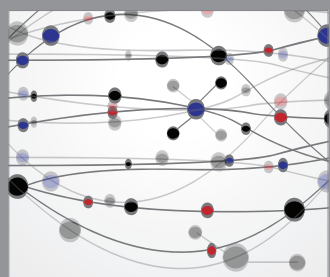

The Scientific World Journal
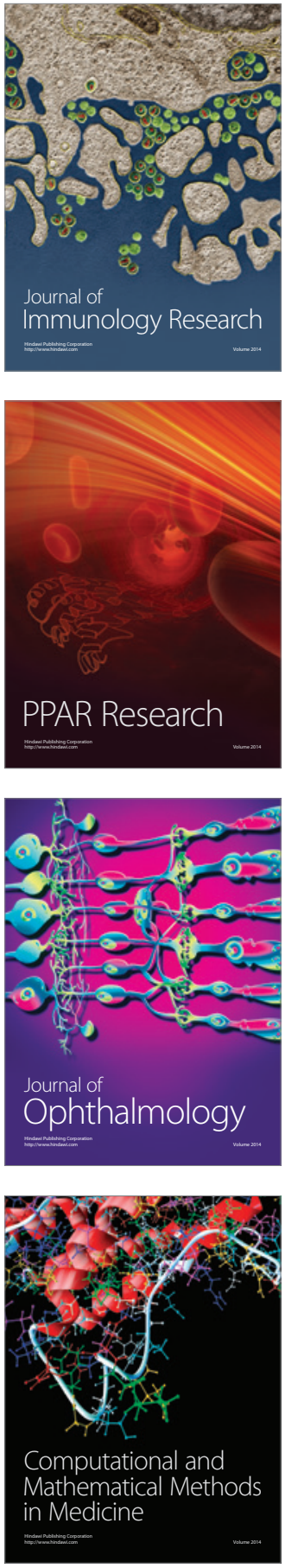

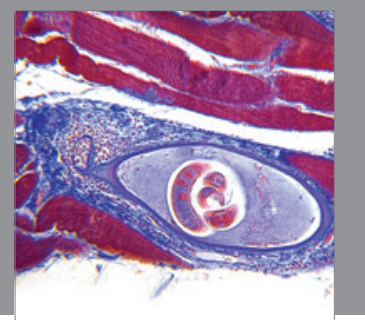

Gastroenterology

Research and Practice
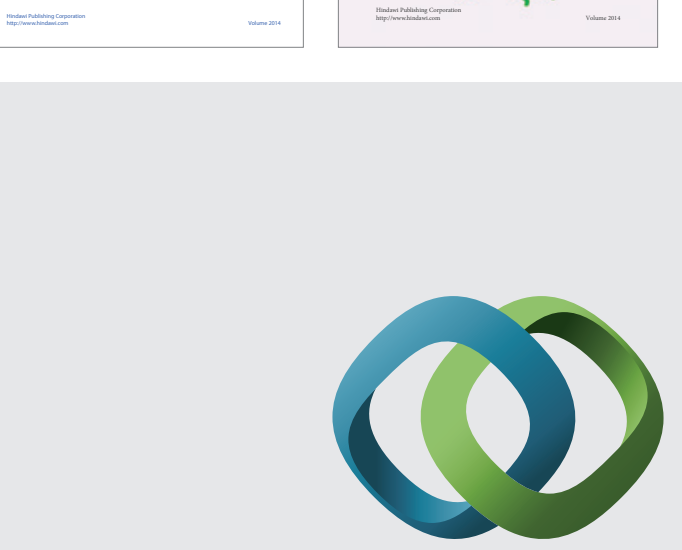

\section{Hindawi}

Submit your manuscripts at

http://www.hindawi.com
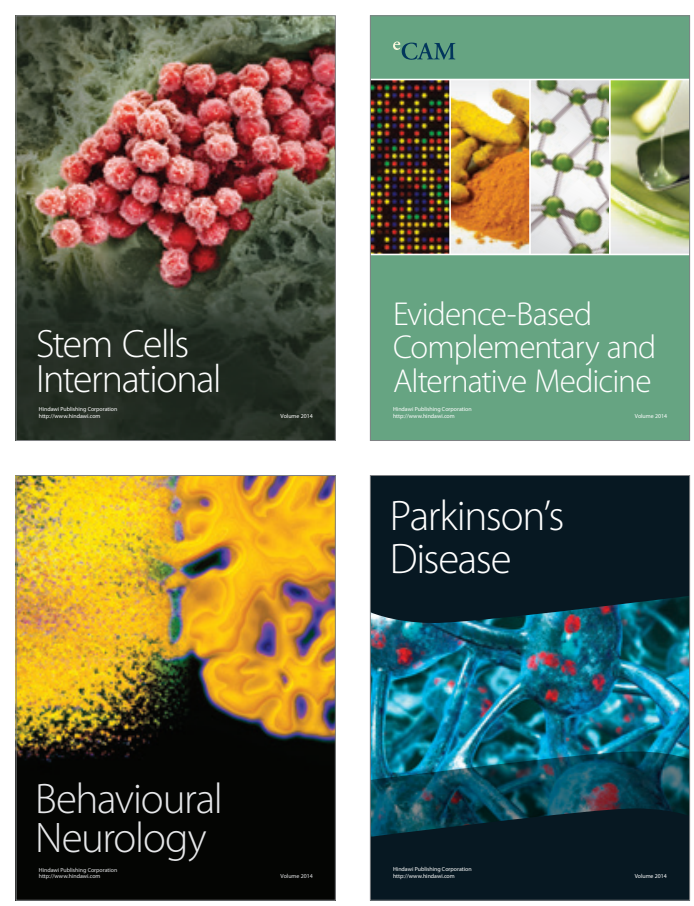

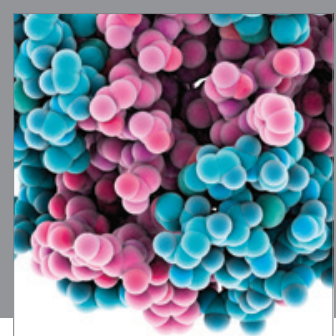

Journal of
Diabetes Research

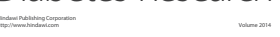

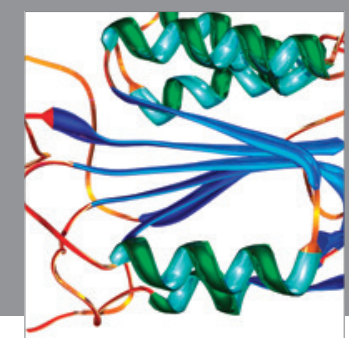

Disease Markers
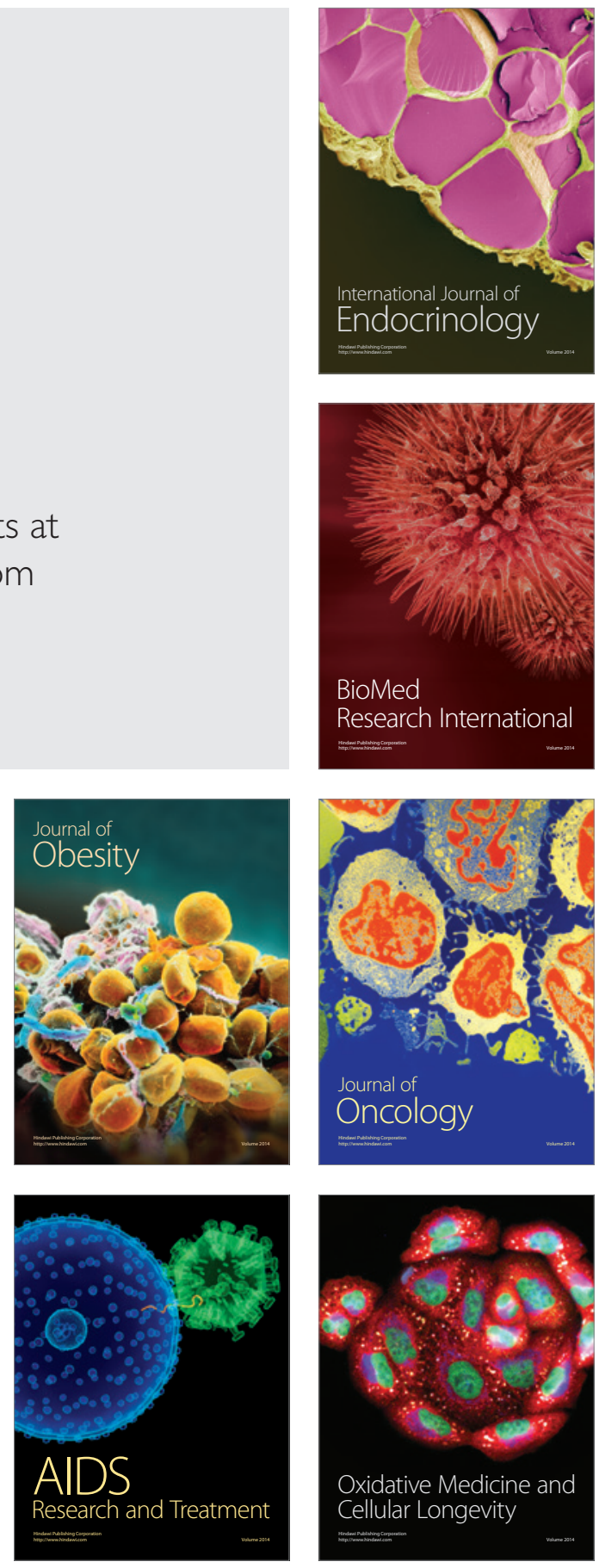